\title{
An exploration of the basis for patient complaints about the oldness of magazines in practice waiting rooms: cohort study
}

The abstract of this Research paper (BMJ 2014;349:g7262, doi:10.1136/bmj.g7262) contains an error in the results 